\title{
Simulating Transient Sediment Waves in Aggraded Alluvial Channels by Double-Decomposition Method
}

\author{
Gokmen Tayfur ${ }^{1}$ and Vijay P. Singh, F.ASCE ${ }^{2}$
}

\begin{abstract}
By using the double-decomposition (DD) method, this study simulates transient sediment waves caused by aggradation described by a diffusion-type partial differential equation (PDE). The DD method solves the PDE by decomposing the solution function for sediment rate into a summation of $M$ number of components, where $M$ stands for the order of approximation. The solution was approximated by considering only the first three terms. The model satisfactorily simulated laboratory-measured aggradation bed profiles with, on average, a mean absolute error (MAE) of $0.70 \mathrm{~cm}$, a root-mean-square error (RMSE) of $0.84 \mathrm{~cm}$, a mean relative error (MRE) of $1.11 \%$, and $R^{2}=0.95$. The model performance was also tested by using numerical and error-function solutions. In addition, the results obtained from application of the DD solution to hypothetical field cases were found to be theoretically compatible with what may be observed in natural streams. However, sediment wave fronts in later periods of the simulation time reached equilibrium bed levels more quickly, around in the middle section of the channel. DOI: 10.1061/(ASCE)HE.1943-5584.0000326. @ 2011 American Society of Civil Engineers.
\end{abstract}

CE Database subject headings: Channels; Sediment transport; Decomposition; Simulation.

Author keywords: Alluvial channel; Transient sediment wave; Diffusion wave; Double decomposition; Sediment transport rate; Sediment concentration; Simulation; Bed profile.

\section{Introduction}

Sediment transport in alluvial channels has been extensively studied both experimentally and mathematically. Experimental studies have involved extensive flume and field observations. Guy et al. (1966) carried out 339 uniform flow and sediment transport experiments from 1956 to 1961 by using a recirculating flume to obtain mean values of the resistance and transport variables for a wide range of conditions most commonly found in natural streams and artificial channels. Soni (1975) conducted aggradation experiments in a laboratory flume subjected to a wide range of sediment loading at the upstream end of the flume and recorded bed elevation changes. Many laboratory flume flow and sediment transport experiments are summarized in Lisle et al. (1997). Field studies of sediment transport have also been conducted by Langbein and Leopold (1968), Wathen and Hoey (1998), and Lisle et al. (2001), among others. Flume experimental and field studies have contributed to the enhanced understanding of basic mechanisms of sediment wave movement in alluvial channels.

Considerable effort has also been devoted to developing theoretical models for predicting sediment movement in alluvial channels. These models have ranged from simple conceptual representations of transport in uniform flow in clear water (Vreugdenhil and de Vries 1973; de Vries 1973) to comprehensive

${ }^{1}$ Professor, Dept. of Civil Engineering, Izmir Institute of Technology, Urla, Izmir, 35340, Turkey. E-mail: gokmentayfur@iyte.edu.tr

${ }^{2}$ Caroline and William N. Lehrer Distinguished Chair and Professor, Dept. of Biological and Agricultural Engineering, and Dept. of Civil and Environmental Engineering, Texas A\&M Univ., College Station, TX 77843-2117 (corresponding author). E-mail: vsingh@tamu.edu

Note. This manuscript was submitted on October 2, 2009; approved on September 23, 2010; published online on September 27, 2010. Discussion period open until September 1, 2011; separate discussions must be submitted for individual papers. This paper is part of the Journal of $\boldsymbol{H y}$ drologic Engineering, Vol. 16, No. 4, April 1, 2011. CASCE, ISSN 10840699/2011/4-362-370/\$25.00. representations treating transport in sediment-laden nonuniform and unsteady flow with interaction between suspended sediment and movable bed layer (Pianese 1994; Wu 2004; Wu et al. 2004; Tayfur and Singh 2006, 2007). Such situations may be encountered during flash floods, landslides, and bank failures. In such situations, not only the sediment transport but also the flow is unsteady and nonuniform, and sediment transport takes place under nonequilibrium conditions. For modeling such situations, the requirement of sophisticated numerical models are inevitable (Singh et al. 2004; Wu et al. 2004; Vasquez et al. 2008; Aricò and Tucciarelli 2008).

Analytical solutions have also been attempted for simulating sediment transport in mostly aggrading channels (de Vries 1965, 1973; Soni 1981a, b; Dietrich et al. 1999; Shan and Hong 2001). An alluvial channel can aggrade under many situations, such as withdrawal of clear water from a stream without any change in other controlling parameters, or increase in sediment supply in excess capacity of the stream to transport (Soni 1981a). Sediment supply (injection) at a section upstream of a channel would cause deposition along the channel length as sediment wave front moves toward downstream. A diffusion-type partial differential equation (PDE) for representing the bed level change in time and space in aggraded channels was developed by de Vries (1973). By using the dynamic equation of motion and the continuity equations for water and sediment, Soni (1981a, b), under some simplifications of these equations, developed a diffusion wave (DW) equation for representing the temporal and spatial change of sediment rate in an aggraded alluvial channel. Assuming an infinite channel length, he obtained an error-function solution for sediment rate by using the Laplace transform. The infinite channel length assumption has been employed in some other studies as well (de Vries 1973; Shan and Hong 2001). Tayfur and Singh (2006) tested their numerical model against the analytical solution of Soni (1981a, b) and showed the overall poor performance of the analytical solution. This may be because of the simplification in the governing equations and perhaps an assumption of the infinite channel length. 
The objective of this study is to introduce a mathematical solution technique of the double-decomposition (DD) method developed by Adomian $(1984,1988)$ for solving the diffusion equation representing the temporal and spatial change in bed level (or sediment rate) in aggraded channels. The method decomposes the solution function for sediment rate into a sum of $M$ number of components, where $M$ stands for order of approximation. As opposed to the error-function solution, the DD method does not impose an infinite channel length constraint. The solution methodology is simple and straightforward. The solution is tested against measured data. The method is further evaluated by simulating bed profiles for several hypothetical scenarios.

\section{Diffusion Wave Model}

\section{Analytical Solutions}

The following DW model for the determination of bed transients in alluvial channels, based on the dynamic equation of motion and the equation of continuity both for sediment and water, was developed by de Vries (1973):

$$
\frac{\partial z}{\partial t}-D \frac{\partial^{2} z}{\partial x^{2}}=0
$$

where $D=$ diffusion coefficient $\left(L^{2 / T}\right)$, which is expressed as (Soni 1981a)

$$
D=\frac{\beta G}{3 S_{o}(1-p)}
$$

where $\beta=$ coefficient whose value is suggested to be 5.0 (Soni 1981a); $G=$ sediment transport rate; $S_{o}=$ bed slope; and $p=$ sediment mass porosity.

An analytical solution of Eq. (1), subjected to the initial condition of $z(x, 0)=0.0$ and boundary conditions of $z(0, t)=z_{o}$ and $z(\infty, t)=0.0$, is given as (Soni 1981a)

$$
z=z_{o} \operatorname{erfc}\left(-\frac{x}{2 \sqrt{D t}}\right)
$$

where $\operatorname{erfc}()=$. complementary error function and is, for any arbitrary variable $y$, defined as (Vreugdenhil and de Vries 1973)

$$
\operatorname{erfc}(y)=\frac{2}{\sqrt{\pi}} \int_{y}^{\infty} e^{-\xi^{2}} d \xi
$$

Taking the shape of the transient bed profile given by Eq. (3), the behavior of variation of $z_{o}$ with time is determined by Soni (1975) as

$$
z_{o}=\frac{\sqrt{\pi} \Delta G t}{2(1-p) \sqrt{D t}}
$$

where $\Delta G=$ excess sediment transport rate, which is the total sediment transport rate $(G)$ minus equilibrium sediment transport rate $\left(G_{e}\right) . G_{e}$ is the rate that is carried by the flow causing no aggradation. To obtain the aggradation wave front movement in the channel, sediment is supplied at the upstream end of a channel at an excess rate of the equilibrium load (Soni 1981a, b).

Soni (1981b), analogous to Eq. (1), developed another version of the DW equation, which is expressed not as a function of bed level $z$, but as a function of sediment transport rate, $G$, as

$$
\frac{\partial G}{\partial t}-K_{o} \frac{\partial^{2} G}{\partial x^{2}}=0
$$

where

$$
K_{o}=\frac{C_{o} g q_{w}(f / 8)^{0.5}}{(1-p)}
$$

where $C_{o}=$ coefficient whose value is determined to be 0.372 (Soni 1981b); $g$ = gravitational acceleration; $q_{w}=$ unit flow discharge; and $f=$ friction coefficient.

Soni (1981b) developed the governing Eq. (6) from the use of the dynamic wave equation of momentum, the resistance equation, the sediment and water continuity equations, and the functional relation of $G=C_{o} U_{*}^{m}$, where $U_{*}=$ shear velocity and $m=$ an exponent whose value is taken as 3 . In his development, Soni assumed a quasi-steady water flow and neglected velocity and flow depth gradient terms in the momentum equation. Soni (1981b) obtained the following analytical solution for Eq. (6) by applying the Laplace transform under the initial condition of $G(x, 0)=G_{e}$ and boundary conditions of $G(0, t)=G_{e}+\Delta G_{e}$ and $G(\infty, t)=G_{e}$ :

$$
G=\Delta G_{e} \operatorname{erfc}\left(-\frac{x}{2 \sqrt{K_{o} t}}\right)+G_{e}
$$

When $G_{e}=0.0$, the solution expressed by Eq. (8) is similar to the solution given by Eq. (3). Also, the analytical solutions given by Eqs. (3) and (8) assume that the channel has an infinite length. Tayfur and Singh (2006) tested their numerical model and the analytical solution against the measured data and showed that the numerical model performed better than the analytical solution.

\section{Double-Decomposition Method}

The equation to be solved by the DD method is the diffusion equation employed by Soni (1981b)

$$
\frac{\partial G}{\partial t}-K_{o} \frac{\partial^{2} G}{\partial x^{2}}=0
$$

subjected to the following initial and boundary conditions:

$$
\begin{gathered}
G(x, 0)=G_{e} \\
G(0, t)=G_{e}+\Delta G \sin (w t) \\
G(L, t)=G_{e}
\end{gathered}
$$

Eq. (10) represents the initial condition of the equilibrium sediment profile in the channel. Eq. (11) stands for the upstream boundary condition, representing an excess sinusoidal sediment loading at the upstream end of the channel. Eq. (12) stands for the downstream boundary condition, which assumes that bed level because of excess sediment loading decreases to the original bed level at this end.

The DD method, developed by Adomian (1984, 1988), decomposes the solution function into a sum of components. Thus, the solution function for the transport rate $G(x, t)$ can be represented as

$$
G=\sum_{m=0}^{M} G_{m}
$$

where $G_{m}=m$ th approximation of $G$ and $m=0,1,2,3, \ldots, M$; and $M=$ arbitrary number indicating the order of approximation. In this study, a three-term approximation is considered to be sufficient, because there may not be a need for including higher-order terms. Employing the operators, $L_{t}=\partial / \partial t$ and $L_{x x}=\partial^{2} / \partial x^{2}$, Eq. (9) can be written as 


$$
L_{t}(G)=K_{o} L_{x x}(G)
$$

Eq. (13), with the use of Eq. (14), can be expressed as

$$
\sum_{m=0}^{M} G_{m}=\sum_{m=0}^{M} W_{x, m}+\frac{1}{K_{0}} L_{x x}^{-1}\left[L_{t} \sum_{m=0}^{M}\left(G_{m}\right)\right]
$$

where $W_{x, m}, m=0,1,2,3, \ldots, M,=$ coefficients.

The first-term approximation can be expressed as

$$
S_{1}(x, t)=G_{0}=W_{0,0}+x W_{0,1}
$$

Applying the first boundary condition [Eq. (11)] yields $W_{0,0}=G_{e}+\Delta G \sin (w t)$ and the second boundary condition [Eq. (12)] yields $W_{0,1}=-\Delta G \sin (w t) / L$. Substituting these coefficients into Eq. (16) yields the first-term approximation solution as

$$
S_{1}(x, t)=G_{0}=G_{e}+\Delta G \sin (w t)\left(1-\frac{x}{L}\right)
$$

The second-term approximation can be expressed as

$$
S_{2}(x, t)=S_{1}(x, t)+G_{1}
$$

where

$$
\begin{gathered}
G_{1}=W_{0,1}+x W_{1,1}+\frac{1}{K_{o}} L_{x x}^{-1}\left[L_{t} G_{o}\right] \\
L_{t} G_{0}=\Delta G w \cos (w t)\left(1-\frac{x}{L}\right) \\
L_{x x}^{-1} L_{t} G_{0}=\iint \Delta G w \cos (w t)\left(1-\frac{x}{L}\right) d x d x \\
=\Delta G w \cos (w t)\left(\frac{x^{2}}{2}-\frac{x^{3}}{6 L}\right)
\end{gathered}
$$

Substituting Eq. (21) into Eq. (19) yields $G_{1}=W_{0,1}+x W_{1,1}+$ $1 / K_{o}\left[\Delta G w \cos (w t)\left(x^{2} / 2-x^{3} / 6 L\right)\right]$. Substitution of this $G_{1}$ into Eq. (18) results in

$$
\begin{aligned}
S_{2}(x, t)= & G_{e}+\Delta G \sin (w t)\left(1-\frac{x}{L}\right)+W_{0,1}+x W_{1,1} \\
& +\frac{1}{K_{o}} \Delta G w \cos (w t)\left(\frac{x^{2}}{2}-\frac{x^{3}}{6 L}\right)
\end{aligned}
$$

The use of the first and second boundary conditions [Eqs. (11) and (12)] yields $W_{0,1}=0.0$ and $W_{1,1}=-\Delta G w L \cos (w t) /\left(3 K_{o}\right)$, respectively. Substitution of these coefficients into Eq. (22) results in the second-term approximation as

$$
\begin{aligned}
S_{2}(x, t)= & G_{e}+\Delta G \sin (w t)\left(1-\frac{x}{L}\right) \\
& +\frac{\Delta G w \cos (w t)}{K_{o}}\left(\frac{x^{2}}{2}-\frac{x^{3}}{6 L}-\frac{x L}{3}\right)
\end{aligned}
$$

$G_{1}$ is equal to the last term on the right-hand side of Eq. (23).

The third-term approximation can be expressed as

$$
S_{3}(x, t)=S_{2}(x, t)+G_{2}
$$

where

$$
G_{2}=W_{0,2}+x W_{1,2}+\frac{1}{K_{o}} L_{x x}^{-1}\left[L_{t} G_{1}\right]
$$

$$
\begin{gathered}
L_{t} G_{1}=\frac{\Delta G w^{2} \sin (w t)}{K_{o}}\left(\frac{x L}{3}-\frac{x^{2}}{2}+\frac{x^{3}}{6 L}\right) \\
L_{x x}^{-1} L_{t} G_{1}=\iint \frac{\Delta G w^{2} \sin (w t)}{K_{o}}\left(\frac{x L}{3}-\frac{x^{2}}{2}+\frac{x^{3}}{6 L}\right) d x d x \\
=\frac{\Delta G w^{2} \sin (w t)}{K_{o}}\left(\frac{L x^{3}}{18}-\frac{x^{4}}{24}+\frac{x^{5}}{120 L}\right)
\end{gathered}
$$

Substituting Eq. (27) into Eq. (25) yields $G_{2}=W_{0,2}+x W_{1,2}+$ $1 / K_{o}\left[\Delta G w^{2} \sin (w t) / K_{o}\left(L x^{3} / 18-x^{4} / 24+x^{5} / 120 L\right)\right]$. Substituting this $G_{2}$ into Eq. (24) results in

$$
\begin{aligned}
S_{3}(x, t)= & G_{e}+\Delta G \sin (w t)\left(1-\frac{x}{L}\right) \\
& +\frac{\Delta G w \cos (w t)}{K_{o}}\left(\frac{x^{2}}{2}-\frac{x^{3}}{6 L}-\frac{x L}{3}\right)+W_{0,2}+x W_{1,2} \\
& +\frac{\Delta G w^{2} \sin (w t)}{K_{o}^{2}}\left(\frac{L x^{3}}{18}-\frac{x^{4}}{24}+\frac{x^{5}}{120 L}\right)
\end{aligned}
$$

Coefficients in Eq. (28) are found by the application of the first and second boundary conditions [Eqs. (11) and (12)] as $W_{0,2}=0.0$ and $W_{1,2}=-\Delta G w^{2} L^{3} \sin (w t) /\left(45 K_{o}^{2}\right)$, respectively. Substitution of these coefficients into Eq. (28) results in the third-term approximation as

$$
\begin{aligned}
S_{3}(x, t)= & G \\
= & G_{e}+\Delta G \sin (w t)\left(1-\frac{x}{L}\right) \\
& +\frac{\Delta G w \cos (w t)}{K_{o}}\left(\frac{x^{2}}{2}-\frac{x^{3}}{6 L}-\frac{x L}{3}\right) \\
& +\frac{\Delta G w^{2} \sin (w t)}{K_{o}^{2}}\left(\frac{L x^{3}}{18}-\frac{x^{4}}{24}+\frac{x^{5}}{120 L}-\frac{x L^{3}}{45}\right)
\end{aligned}
$$

Eq. (29) is the DD solution for the PDE, expressed by Eq. (9). Hence, by Eq. (29), one can obtain the solution for spatial and temporal variation of sediment rate in the channel as a result of sinusoidal loading at the upstream end.

The rate of deposition can be obtained by using the sediment continuity equation:

$$
(1-p) \frac{\partial z}{\partial t}+\frac{\partial G}{\partial x}=0
$$

Differentiating Eq. (29) with respect to $x$ once, and then integrating the resulting expression from $t_{1}$ to $t_{2}$, one can obtain the following expression for computing temporal and spatial variation of the bed level in a channel as a result of excess sinusoidal loading at the upstream end:

$$
\begin{aligned}
z_{t 2}= & z_{t 1}+\frac{1}{1-p}\left\{\frac{\Delta G}{L w}\left[\cos \left(w t_{1}\right)-\cos \left(w t_{2}\right)\right]\right. \\
& \left.+\frac{\Delta G}{K_{0}}\left[\sin \left(w t_{1}\right)-\sin \left(w t_{2}\right)\right]\left(x-\frac{x^{2}}{2 L}-\frac{L}{3}\right)\right\} \frac{1}{(1-p)} \\
& \times\left\{\frac{\Delta G w}{K_{o}^{2}}\left[\cos \left(w t_{2}\right)-\cos \left(w t_{1}\right)\right]\left(\frac{x^{2} L}{6}-\frac{x^{3}}{6}+\frac{x^{4}}{24 L}-\frac{L^{3}}{45}\right)\right\}
\end{aligned}
$$

From Eq. (31), one can obtain the change in bed level in a period of $\Delta t=t_{2}-t_{1}$ at any section of the channel length as a result of 
the sediment movement in channel because of excess sediment loading at the upstream end of the channel.

\section{Model Application}

\section{Flume Sediment Transport Experiments}

The DD solution [Eq. (31)] was tested against the experimental data of aggradation depths measured by Soni $(1975,1981 \mathrm{a}, \mathrm{b})$ in laboratory flume experiments. The recirculatory tilting flume of rectangular cross section was $30.0 \mathrm{~m}$ long, $0.20 \mathrm{~m}$ wide, and $0.50 \mathrm{~m}$ deep. The flume was filled with sand to a depth of $15 \mathrm{~cm}$, which was the equilibrium bed level depth. The sand forming the equilibrium bed and the injected excess sediment had a median sieve diameter of $d_{50}=0.32 \mathrm{~mm}$ and a specific gravity of $2.65 \mathrm{~g} / \mathrm{cm}^{3}$. The sediment was dropped manually at the upstream section at a constant rate in excess of the equilibrium concentration to cause aggradation. Aggradation runs were continued until the endpoint of the transient profiles reached the downstream end. The aggradation runs were conducted by using two flow discharges of 4 and $7 \mathrm{~L} / \mathrm{s}$, slopes ranging from 0.00212 to 0.00652 , and with an overloading varying from 0.3 to $4.0 G_{e}$, where $G_{e}$ is the average equilibrium sediment rate. The details of the experiments can be obtained in Soni (1981a).

Soni $(1975,1981 a, b)$ loaded a constant excess sediment rate during each experiment. Because the DD solution requires a sinusoidal loading, the excess loading is represented by a half sinusoidal curve while keeping the total amount constant (Fig. 1). For example, for $G_{e}=1.66 \times 10^{-5} \mathrm{~m}^{2} / \mathrm{s}$, the excess load of $1.35 G_{e}=$ $2.24 \times 10^{-5} \mathrm{~m}^{2} / \mathrm{s}$. That means, every second, there is an excess loading of $2.24 \times 10^{-5} \mathrm{~m}^{2} / \mathrm{m}$ volume of sediment at the upstream end. In $120 \mathrm{~min}$, for example, the total loading becomes $0.1614 \mathrm{~m}^{3} / \mathrm{m}$ This excess volume is loaded into the DD solution in $120 \mathrm{~min}$ by representing it as a half sine curve as presented in Fig. 1. According to Fig. 1, the period $w=\pi / 7,200$ and, hence, loading is zero at $t=0$ and $t=7,200 \mathrm{~s}$, and it reaches a maximum at $t=3,600 \mathrm{~s}$, where the loading is equal to $\Delta G . \Delta G$ can be obtained from $\int_{0}^{7,200} \Delta G \sin (w t) d t=0.1614 \mathrm{~m}^{3} / \mathrm{m}$, which results in $\Delta G=3.65 \times 10^{-5} \mathrm{~m}^{3} / \mathrm{m}$.

Figs. 2-5 present the simulation of bed profiles at 15, 45, 75, and 105 min of the experiment, respectively, by the DD solution for the case where excess loading is equal to $1.35 G_{e}$, where $G_{e}=$ $16.6 \times 10^{-6} \mathrm{~m}^{2} / \mathrm{m}$ (Soni $1975,1981 \mathrm{a}, \mathrm{b}$ ). The porosity is assumed to be 0.45 . From the available information, one can find the shear velocity, $u_{*}=\sqrt{g R S_{o}}$ is equal to $0.032 \mathrm{~m} / \mathrm{s}$, where $R=$ the hydraulic radius. Then, from $f=8 u_{*}^{2} / u^{2}$, one can obtain the value of the friction coefficient as $f=0.174$, where $u=$ the flow velocity. Then, from $K_{o}=C_{o} g q_{w}(f / 8)^{0.5} /(1-p)$, one can obtain the value of $K_{o}=0.02$, which is in agreement with Soni (1981b).

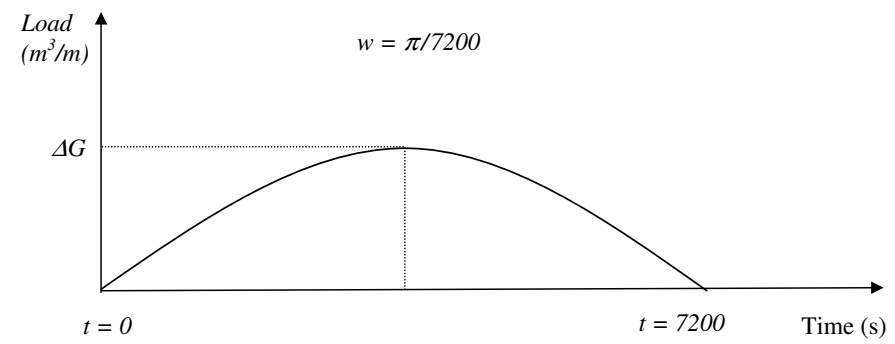

Fig. 1. Schematic representation of a half-sine function for sediment loading
Also, shown in those figures are comparisons against the errorfunction solution [Eq. (3)] and the numerical solution (Tayfur and Singh 2006). The error-function solution employs $G(\infty, t)=G_{e}$, i. e., as the channel is sufficiently long, the aggradation profile would reach the original bed level. This is analogous to the boundary conditions which are often employed in groundwater flow and contaminant transport problems. The numerical solution solves the system of flow continuity and momentum and sediment continuity equations. It approximates the momentum equation by the kinematic wave approximation, i.e., it ignores the convective and local acceleration terms in the momentum equation. For sediment transport function, it employs the kinematic wave theory model developed by Tayfur and Singh (2006). It also employs the Dietrich (1982) formulation for particle fall velocity and the Bridge and Dominic (1984) model for particle velocity. It relates suspended sediment concentration to flow variables and particle characteristics through the Velikanov (1954) relation. It solves the system of equations by the Lax explicit finite difference method. The details of the numerical model can be obtained in Tayfur and Singh (2006). The comparison of the DD solution with the numerical solution may not, at first, seem appropriate, because each employs a different mathematical model. However, Bor (2009) tested the kinematic wave model against the DW and presented that both models yielded almost the same results. Furthermore, the purpose here is to test the DD solution against different solutions and models.

Fig. 2 shows the simulation at the 15 th minute of the experiment. The measured level reaches the equilibrium bed at the third meter, and then fluctuates significantly. The sediment wave front reaches the equilibrium level earlier at the second meter in the case of the numerical model, but at the fifth meter in the DD solution. In the case of error-function solution, it never reaches the original level. Fig. 3 shows the bed profile simulation at the 45 th minute. The measured profile reaches the equilibrium bed level around the 10th meter, although it fluctuates from that

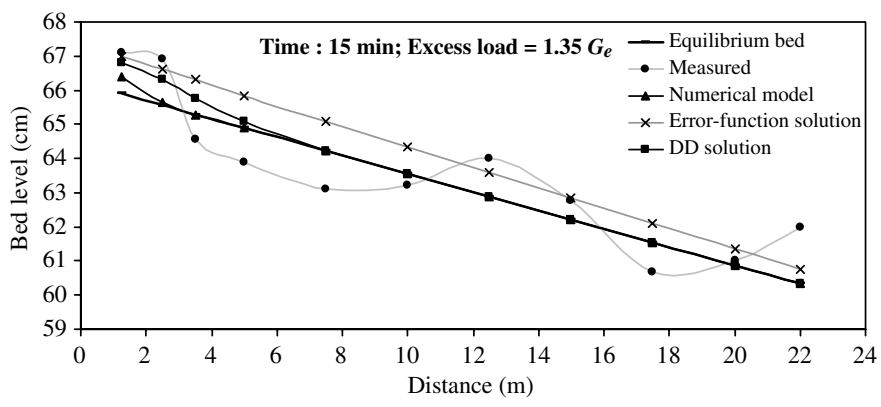

Fig. 2. Simulation of bed profile at 15 th minute of the experiment

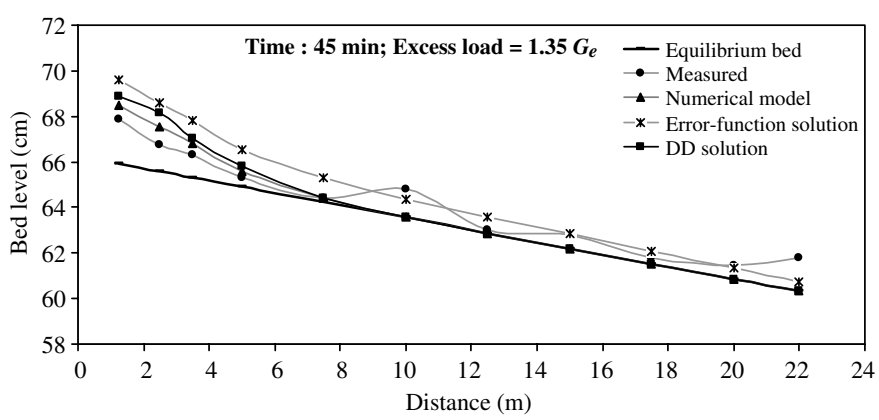

Fig. 3. Simulation of bed profile at 45 th minute of the experiment 
location onward. The numerical model and DD solutions closely follow the measured profile until the 10th meter. The error-function solution, although, on average, follows the measured profile, in the first $10 \mathrm{~m}$, it overpredicts. However, after the 10th meter, it shows better performance than others in capturing the measured bed levels. Fig. 4 shows the simulation at the 75 th minute of the experiment. As seen, the measured profile gradually reaches the original level at around the 15th meter, whereas the numerical model reaches that level at the 12th meter, and DD model at the 10th meter, both closely following the measured profile. The error-function solution reaches the original level at a later distance of the 18th meter. It significantly overpredicts the measured data, especially in the first 14-m distance. Fig. 5 shows the simulation at the 105 th minute of the experiment. The sediment wave front reaches the equilibrium bed level at an earlier distance, around the 12th meter in the DD solution. The numerical model shows better performance in capturing the measured data. The error-function solution overpredicts through the end section of the flume. The overall computed mean absolute error (MAE), root mean square error (RMSE), mean relative error (MRE), and $R^{2}$ for all the models, considering all the 4 simulations in Figs. 2-5 are summarized in Table 1. Compared with the DD and numerical models, the error-function solution produced high error values of MRE, MAE, and RMSE and low $R^{2}=0.813$. The DD and the numerical models predicted the measured bed levels with less than $2 \%$ error: MAE of $0.70 \mathrm{~cm}$ and RMSE of $0.80 \mathrm{~cm}$. They produced similar performance with $R^{2}=0.93$.

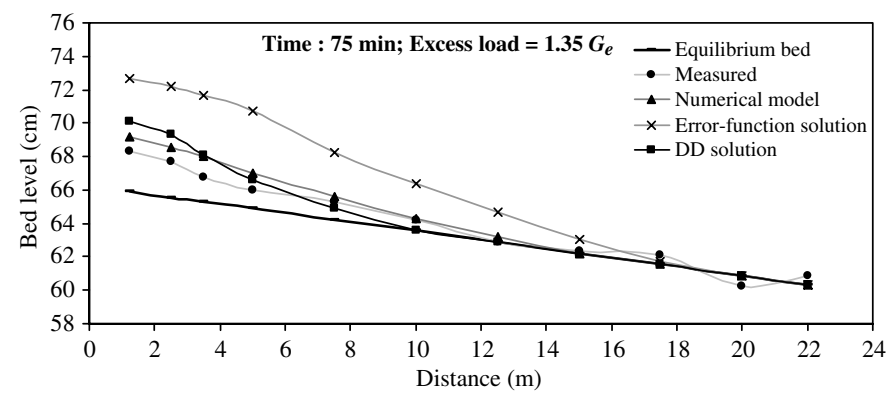

Fig. 4. Simulation of bed profile at 75th minute of the experiment

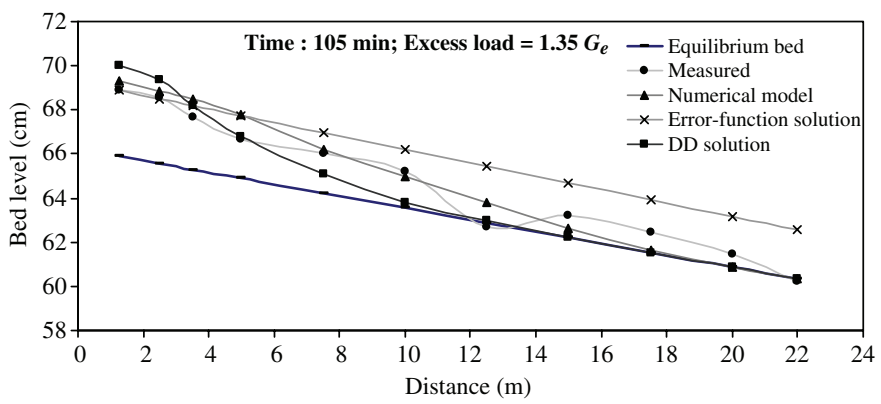

Fig. 5. Simulation of bed profile at 105th minute of the experiment

Table 1. Error Measures for the Three Model [Experiment I: Excess load $=1.35 G_{e}$ ]

\begin{tabular}{lcccc}
\hline MODEL & MAE $(\mathrm{cm})$ & MRE $(\%)$ & RMSE $(\mathrm{cm})$ & $R^{2}$ \\
\hline Numerical solution & 0.65 & 1.01 & 0.765 & 0.936 \\
Error-function solution & 1.39 & 2.15 & 1.876 & 0.813 \\
DD solution & 0.75 & 1.16 & 0.887 & 0.928 \\
\hline
\end{tabular}

Figs. 6-8 present the simulation of bed profiles at 30,60 , and $90 \mathrm{~min}$ of another experiment, respectively, by the models for the case where excess loading is equal to $90 \%$ of the $G_{e}$, where $G_{e}=34.4 \times 10^{-6} \mathrm{~m}^{2} / \mathrm{s}$ (Soni 1975, 1981a, b). Fig. 6 shows the simulation at the 30 th minute of the experiment. As is seen, the DD solution closely follows the measured data and both reach the original bed level at the 10th meter of the distance, although the measured profile fluctuates afterward. The numerical model reaches the original bed level earlier, at the eighth meter of the distance, whereas the error-function solution never reaches the original level. Fig. 7 shows the simulation at the 60th minute of the experiment. According to Fig. 7, the measured profile reaches the original level at the 18th meter and then fluctuates slightly afterward. The numerical and DD solutions show the same profiles, following the measured profile, although both reach the original level earlier at the 15 th meter of the distance. Although the error-function solution never reaches the original level, it shows better performance overall in predicting the measured bed levels. Fig. 8 shows the simulation at the 90th minute of the experiment. Although the wave front in the case of the error-function solution never reaches the original bed level, it overall better predicts measured bed levels,

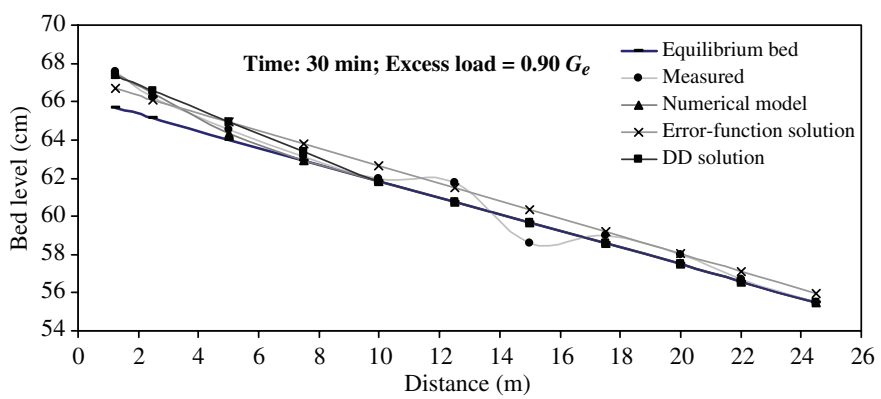

Fig. 6. Simulation of bed profile at 30th minute of the experiment

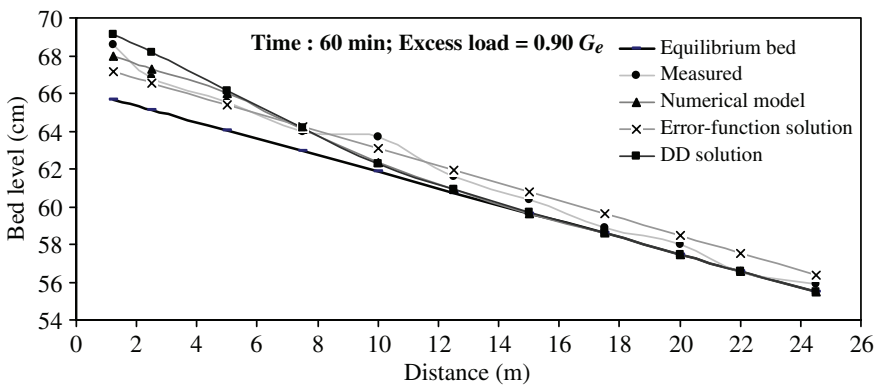

Fig. 7. Simulation of bed profile at 60 th minute of the experiment

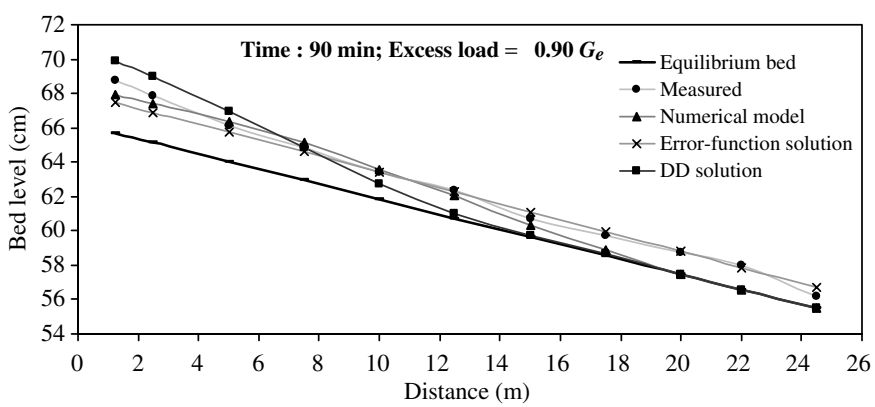

Fig. 8. Simulation of bed profile at 90th minute of the experiment 
Table 2. Error Measures for the Three Model [Experiment II: Excess load $=0.90 G_{e}$ ]

\begin{tabular}{lcccc}
\hline MODEL & MAE $(\mathrm{cm})$ & MRE $(\%)$ & RMSE $(\mathrm{cm})$ & $R^{2}$ \\
\hline Numerical solution & 0.500 & 0.82 & 0.628 & 0.983 \\
Error-function solution & 0.498 & 0.80 & 0.643 & 0.985 \\
DD solution & 0.658 & 1.06 & 0.793 & 0.980 \\
\hline
\end{tabular}

especially in the second half of the channel. The wave fronts in the numerical and DD solutions reach the original bed level earlier, at the 20th and 16th meters, respectively. The overall computed MAE, RMSE, MRE, and $R^{2}$ for all the models, considering all these three simulations in Figs. 6-8 are summarized in Table 2. On the average, all the models produced comparable errors.

\section{Hypothetical Case Application}

The DD solution is investigated for a hypothetical channel having a 200-m length, a 20-m width, and a bed slope of 0.0025 . It is assumed that flow rate $Q=20 \mathrm{~m}^{3} / \mathrm{s}$, Chezy coefficient $C_{z}=20 \mathrm{~m}^{0.5} / \mathrm{s}$, and porosity $p=0.40$. A constant sediment loading of $10 \mathrm{t} / \mathrm{h}$ is assumed at the upstream end of the channel.

Fig. 9 shows the simulation of bed profiles at different times of the simulation. As time progresses bed level increases along the channel length. Fig. 10 shows the bed profile simulation at the 30th minute, under different sediment loadings. As the loading increases, bed levels increase along the channel length. Fig. 11 shows the simulation of bed profiles along the channel under the same sediment loadings of $10 \mathrm{t} / \mathrm{h}$ but different $K_{o}$ values. Different $K_{o}$ values imply different flow conditions. The higher is $K_{o}$ value, the higher the flow discharge becomes. Under higher flow discharges, the bed levels are low. This is because a high flow rate carries the sediment faster downstream of the channel, thus resulting in low bed levels. These results imply that the model

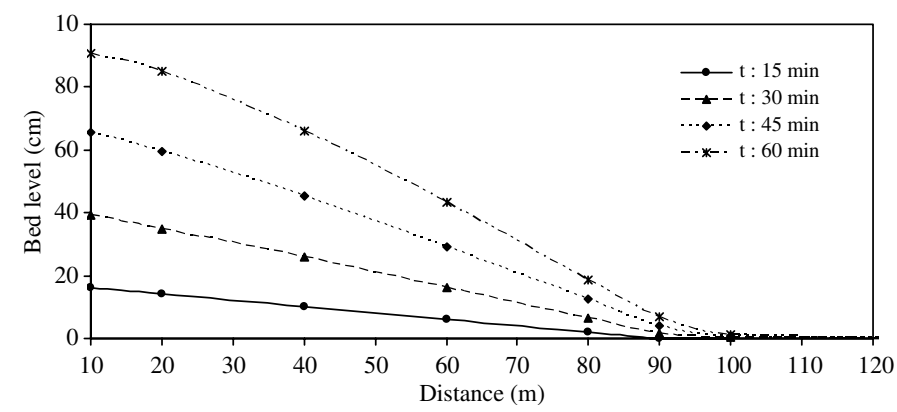

Fig. 9. Simulation of bed profile along the hypothetical channel at different times of the simulation period

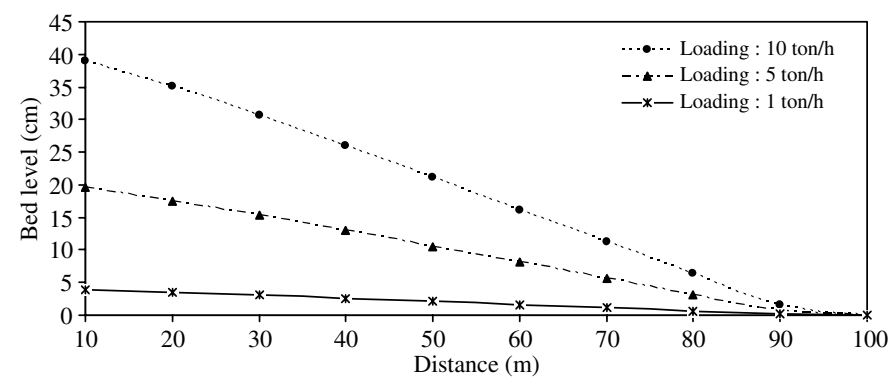

Fig. 10. Simulation of bed profile at the 30th minute along the hypothetical channel under different sediment loadings

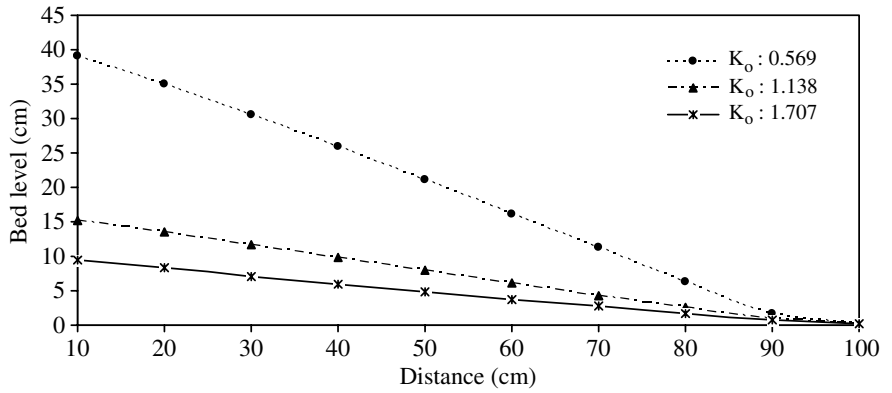

Fig. 11. Simulation of bed profile at the 30th minute along the hypothetical channel under different $K_{o}$ values

can produce results compatible with those that one may observe in the field.

\section{Order of Magnitude Analysis}

Simulation results of actual experimental data and hypothetical case studies, summarized previously, show that sediment wave fronts in the DD solution reach original bed levels quicker at around middle sections of the channel in later periods of simulation. This may be attributable to the nature of the sinusoidal sediment loading. The DD solution expressed by Eq. (31) contains trigonometric functions of sines and cosines multiplied by the $x$-distance terms. Figs. 12-16 show the change in the magnitude of terms in time at $x=L / 8, x=L / 4, x=L / 2, x=3 L / 4$, and $x=L$ locations, respectively. Term 1 and Term 2 refer to the first and second terms, respectively, within the first bracket on the right-hand side of Eq. (31). Term 3 refers to the term within the last bracket on the right-hand side of Eq. (31). As seen in these figures, Term 1 always has positive values at each location during the simulation period. Term 2, which contains sine terms, produces positive values in the first half period and then negative values in the second half

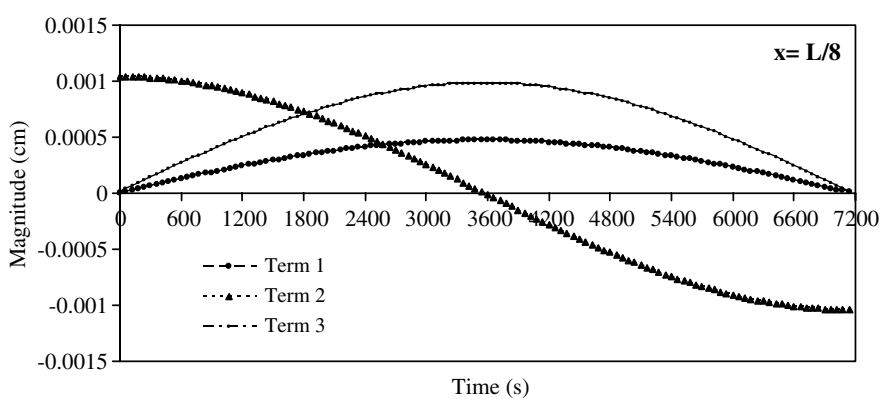

Fig. 12. Change in the magnitude of the terms in time at $x=L / 8$

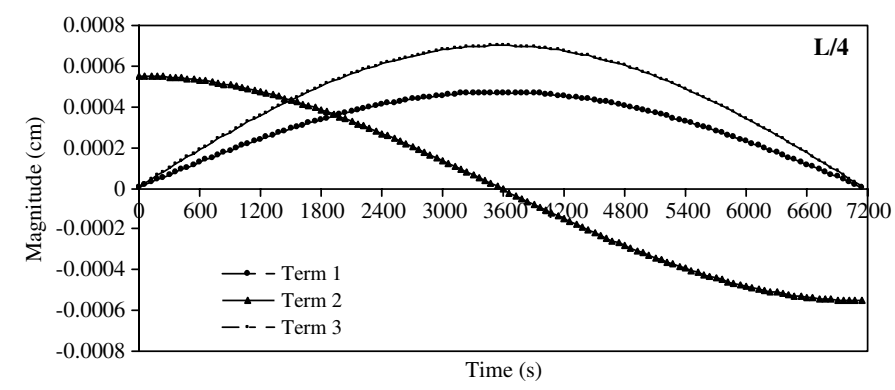

Fig. 13. Change in the magnitude of the terms in time at $x=L / 4$ 


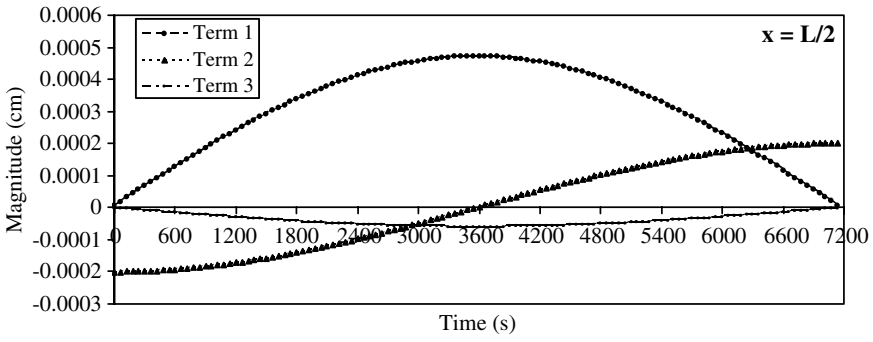

Fig. 14. Change in the magnitude of the terms in time at $x=L / 2$

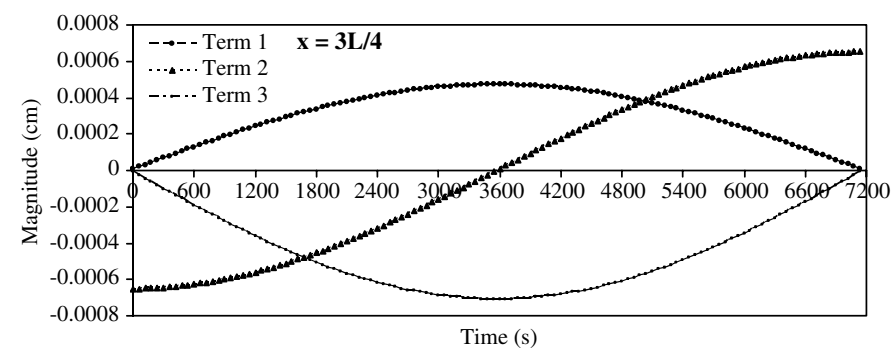

Fig. 15. Change in the magnitude of the terms in time at $x=3 L / 4$

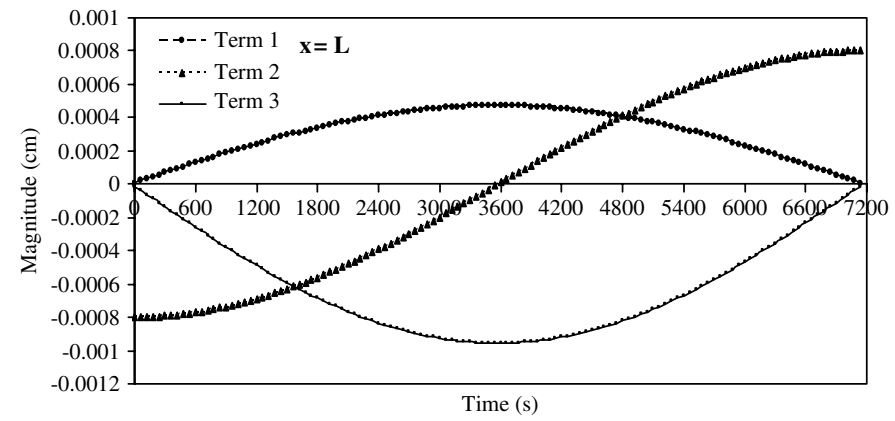

Fig. 16. Change in the magnitude of the terms in time at $x=L$

period of simulation until the middle section of the channel. It shows the same but opposite behavior after the middle section of the channel (see Figs. 12-16). Term 3, after the $x=L / 2$ location toward downstream, constantly produces negative values for all the period of the simulation (see Fig. 14). The magnitude of these negative values increases considerably after location $x=L / 2$ toward the end of the channel. This term may be the responsible one causing faster wave fronts.

\section{Comparison of Order of Approximations}

As stated earlier, this study employed a third-order approximation. To justify this order, a comparison among the order of approximations is performed in this section. This comparison analysis also involves the fourth-order approximation. Eq. (31) is the thirdorder approximation for bed level. As previously mentioned, the third-order approximation for sediment rate was first derived [Eq. (29)] and then, by using the conservation of mass equation [Eq. (30)], the third-order approximation for bed level was obtained [Eq. (31)]. Also, given previously are the first-order and secondorder approximations for sediment rate, as expressed by Eqs. (17) and (23), respectively. By using Eq. (30), one can obtain the firstorder and second-order approximations for bed level as well. The derived first-order approximation would be the cancellation of last

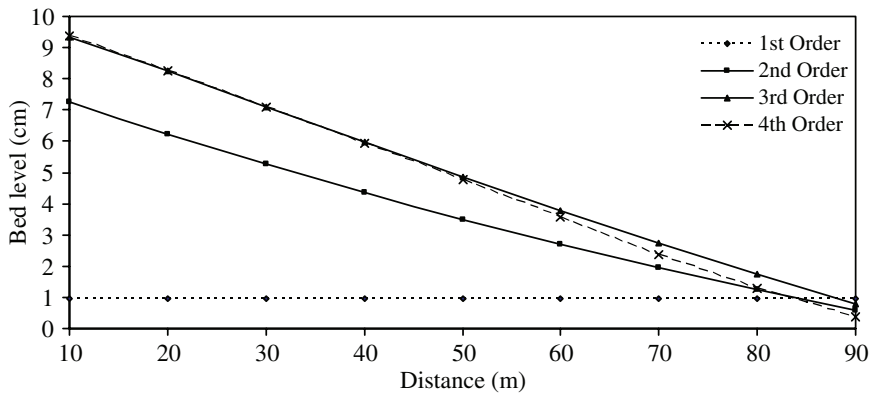

Fig. 17. Comparison of order of approximations at the simulation of 30 th minute $\left[K_{o}=1.707\right.$, excess load $\left.=10 \mathrm{t} / \mathrm{h}\right]$

term and the second term within the first bracket on the right-hand side of Eq. (31) and the second order approximation would be the cancellation of the last term on the right-hand side of Eq. (31). The fourth-order approximation can be obtained, in a similar fashion, by following the same procedure given in the section, "DoubleDecomposition Method." For the sake of brevity, the details of this derivation are not given here. The fourth-order approximation can be obtained as follows:

$$
\begin{aligned}
z_{t 2}= & z_{t 1}+\frac{1}{1-p}\left\{\frac{\Delta G}{L w}\left[\cos \left(w t_{1}\right)-\cos \left(w t_{2}\right)\right]\right. \\
& \left.+\frac{\Delta G}{K_{0}}\left[\sin \left(w t_{1}\right)-\sin \left(w t_{2}\right)\right]\left(x-\frac{x^{2}}{2 L}-\frac{L}{3}\right)\right\}+\frac{1}{(1-p)} \\
& \times\left\{\frac{\Delta G w}{K_{o}^{2}}\left[\cos \left(w t_{2}\right)-\cos \left(w t_{1}\right)\right]\left(\frac{x^{2} L}{6}-\frac{x^{3}}{6}+\frac{x^{4}}{24 L}-\frac{L^{3}}{45}\right)\right\} \\
& +\frac{1}{(1-p)}\left\{\frac{\Delta G w^{2}}{K_{o}^{3}}\left[\sin \left(w t_{1}\right)-\sin \left(w t_{2}\right)\right]\right. \\
& \left.\times\left(\frac{x^{4} L}{72}-\frac{x^{5}}{120}+\frac{x^{6}}{720 L}-\frac{L^{3}}{840}\right)\right\}
\end{aligned}
$$

Fig. 17 shows the comparison among the order of approximations at the 30th minute of the simulation period for the case where the excess loading is $10 \mathrm{t} / \mathrm{h}$ and $K_{o}=1.707$. As seen in Fig. 17, the first-order approximation produces a constant profile having low magnitude, which is not physical, because its solution is independent of distance $(x)$. By the second-order and third-order approximations, sediment wave fronts with decreasing bed levels move downstream, which is the physical case. Because the DD method decomposes the solution function into summation of a number of components, the higher is the order of approximation, the better the approximation is expected. According to Fig. 17, there is a significant difference between second-order and third-order approximations in terms of magnitudes. Therefore, one has to prefer the third-order approximation. According to Fig. 17, the fourthorder and third-order approximations produced almost the same results (Fig. 17). That means increasing the order after the third one may not significantly alter the results. Therefore, the third order of approximation is sufficient for this particular problem.

\section{Limitations of the Double-Decomposition Solution}

Although the DD solution is found to be satisfactory for the problem addressed in this study and is simple to use, it has its own drawbacks. First, the sediment loading at the upstream end should be sinusoidal. That means that the DD approach may not be useful for cases with different boundary conditions. Second, the method is not able to represent the mechanism of flow-sediment interaction in sediment transport in alluvial channels, because it treats the flow part as steady and uniform. Third, it is not able to treat the sediment 
transport in two phases of water flow which may contain suspended sediment and bed layer. Hence, it is not appropriate for modeling sediment transport of suspended and bed load under nonequilibrium conditions. Fourth, it would be further difficult to extend the DD method to a multidimensional simulation model for modeling sediment transport and bed profiles in two and three dimensions. Hence, its applicability is limited. Nevertheless, the model is easy and straightforward and therefore can be employed for predicting aggraded bed profiles in alluvial channels. Furthermore, it can easily predict sediment rates that can, in turn, be used to design hydraulic structures, and, as such, the method is applicable and can be useful to the profession.

\section{Concluding Remarks}

This study develops a DD solution for simulating bed profiles in aggraded alluvial channels. The method decomposes the solution function into a sum of a number of components. By the DD method, the solution for sediment rate is first derived and then, by using the conservation of mass equation for sediment, the solution for bed level is obtained. By using Eqs. (29) and (31), one can compute the temporal and spatial variation of sediment rate bed levels in alluvial channels, respectively. The model is tested against laboratory bed-level data and hypothetical field cases. It shows a good performance in predicting the measured bed profiles and produces results that are compatible with those that may be observed in natural channels. The satisfactory performance of the solution and the comparison analysis of the order of approximations imply that considering only the first three terms of the series solution (thirdorder approximation) is sufficient for this particular problem.

The performance of the model against the numerical and analytical (error-function) solutions is satisfactory for simulating the experimental data. It shows nearly the same performance as the numerical model, but it is mostly better than the analytical solution. Contrary to the error-function solution, the developed model does not impose any constraint on the solution, such as the infinite channel length.

The order of magnitude analysis results show that the terms within the brackets in Eq. (31) have comparable magnitudes. However, after $x=L / 2$, the third term produces negative values, thus causing faster wave fronts at later times of the simulation period. This is the limitation of the model. In other words, the model is not satisfactorily able to predict bed levels after the middle section of the channel at later periods of simulation.

\section{Acknowledgments}

This research was supported by the Scientific and Technological Research Council of Turkey [TUBITAK] under Grant No. 106M274. The writers extend their sincere appreciation to TUBITAK for the financial support. The writers would also like to express their thanks to Professor Engin Aktas of the Civil Engineering Dept., Izmir Institute of Technology; and Professor Oguz Yilmaz of the Dept. of Mathematics, Izmir Institute of Technology for valuable discussions.

\section{Notation}

The following symbols are used in this paper:

$C_{o}=$ coefficient whose value is determined to be 0.372 ;

$C_{z}=$ Chezy coefficient;

$D=$ diffusion coefficient;

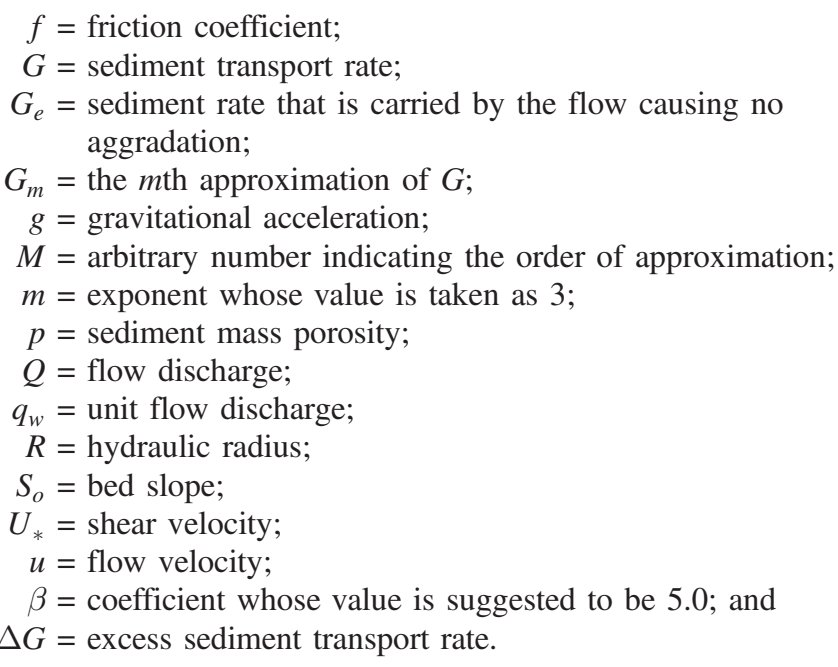

\section{References}

Adomian, G. (1984). "A new approach to nonlinear partial differential equations." J. Math. Anal. Appl., 102, 420-434.

Adomian, G. (1988). "A review of decomposition method in applied mathematics." J. Math. Anal. Appl., 135, 501-544.

Aricò, C., and Tucciarelli, T. (2008). "Diffusive modelling of aggradation and degradation in artificial channels." J. Hydraul. Eng., 134(8), 1079-1088.

Bor, A. (2009). "Numerical modelling of unsteady and non-equilibrium sediment transport in rivers." M.Sc. thesis, Izmir Institute of Technology, Turkey.

Bridge, J. S., and Dominic, D. F. (1984). "Bed load grain velocities and sediment transport rates." Water Resour. Res., 20(4), 476-490.

de Vries, M. (1965). "Consideration about non-steady bed-load transport in open channels." Proc. XI Congress, IAHR, Delft, the Netherlands, 3 , paper 3.8.

de Vries, M. (1973). "River bed variation-Aggradation and degradation." Int. Seminar on Hydraulics of Alluvial Streams, IAHR, Delft, the Netherlands (also available as Delft Hydraulic Laboratory, Delft, 1973, Pub. No. 107).

Dietrich, C. R., Green, T. R., and Jakeman, A. J. (1999). "An analytical model for sediment transport: Application to Murrey and Murrunbridge river reaches, Australia." Hydrol. Processes, 13(5), 763-776.

Dietrich, W. E. (1982). "Settling velocity of natural particles." Water Resour. Res., 18(6), 1615-1626.

Guy, H. P., Simons, D. B., and Richardson, E. V. (1966). "Summary of alluvial channel data from flume experiments, 1956-1961." Professional Paper, 462-I, U.S. Geological Survey 96.

Langbein, W. B, and Leopold, L. B. (1968). "River channel bars and dunes -Theory of kinematic waves." Professional Paper, 422-L, U.S. Geological Survey 20.

Lisle, T. E., Cui, Y. T., Parker, G., Pizzuto, J. E., and Dodd, A. M. (2001). "The dominance of dispersion in the evolution of bed material waves in gravel-bed rivers." Earth Surf. Processes Landforms, 26, 1409-1420.

Lisle, T. E., Pizzuto, J. E., Ikeda, H., Iseya, F., and Kodama, Y. (1997). "Evolution of a sediment wave in an experimental channel." Water Resour. Res., 33, 1971-1981.

Pianese, D. (1994). "Comparison of different mathematical models for river dynamics analysis." Int. Workshop on Floods and Inundations Related to Large Earth Movements. Ministry of Public Works, Rome, paper no. 782, 24.

Shan, Y. X., and Hong, W. O. W. (2001). "The analytical solution for sediment reaction and diffusion equation with generalized initial boundary conditions." Appl. Math. Mech., 22(4), 404-408.

Singh, A. K., Kothyari, U. C., and Raju, K. G. R. (2004). "Rapidly varying transient flows in alluvial rivers." J. Hydraul. Res., 42(5), 473-486. 
Soni, J. P. (1975). "Aggradation in streams due to increase in sediment load." Ph.D. thesis, Univ. of Roorkee, Roorkee, India.

Soni, J. P. (1981a). "An error function solution of sediment transport in aggradation channels." J. Hydrol. (Amsterdam), 49, 107-119.

Soni, J. P. (1981b). "Unsteady sediment transport law and prediction of aggradation parameters." Water Resour. Res., 17(1), 33-40.

Tayfur, G., and Singh, V. P. (2006). "Kinematic wave model of bed profiles in alluvial channels." Water Resour. Res., 42(6), W06414.

Tayfur, G., and Singh, V. P. (2007). "Kinematic wave model for transient bed profiles in alluvial channels under nonequilibrium conditions." Water Resour. Res., 43, W12412.

Vasquez, J. A., Steffler, P. M., and Millar, R. G. (2008). "Modeling bed changes in meandering rivers using triangular finite elements." J. Hydraul. Eng., 134(9), 1348-1352.
Velikanov, M. A. (1954). "Gravitational theory of sediment transport." J. Sci. Soviet Union, Geophys., 4 (in Russian).

Vreugdenhil, C. B., and de Vries, M. (1973). "Analytical approaches to non-steady bedload transport." Research Rep., S 78 part IV, Delft Hydraulic Laboratory, Delft, the Netherlands, 16.

Wathen, S. J., and Hoey, T. B. (1998). "Morphological controls on the downstream passage of a sediment wave in a gravel-bed stream." Earth Surf. Processes Landforms, 23, 715-730.

Wu, W. (2004). "Depth-averaged two-dimensional numerical modelling of unsteady flow and nonuniform sediment transport in open channels." J. Hydraul. Eng., 130(10), 1013-1024.

Wu, W., Vieira, D. A., and Wang, S. S. Y. (2004). "One-dimensional numerical model for nonuniform sediment transport under unsteady flows in channel networks." J. Hydraul. Eng., 130(9), 914-923. 